\title{
Comparison between Preoperative Loading Dose of Ticagrelor versus Clopidogrel on Myocardial Perfusion in Type II Diabetic Patients Presenting with Anterior Wall Myocardial Infarction
}

\author{
Emad Effat Fakhry, Adel Mohammed El Etriby, Ahmed Elmahmoudy Nayel \\ Department of Cardiology, Faculty of Medicine, Ain Shams University, Cairo, Egypt \\ ORCID: \\ Emad Effat Fakhry: https://orcid.org/0000-0002-8993-4250 \\ Adel Mohammed El Etriby: https://orcid.org/0000-0002-8788-4771 \\ Ahmed Mohammed Mahmoudy: https://orcid.org/0000-0001-5604-3104
}

\section{Abstract}

Background: Diabetes mellitus is recognized as an independent risk factor for the development of Coronary No Reflow. Ticagrelor has a faster onset of action and a stronger antiplatelet effect as compared to clopidogrel. The aim of this study is to compare between ticagrelor and clopidogrel loading doses before primary percutaneous coronary intervention (PCI) in Type II diabetic patients presenting with anterior wall ST-Segment Elevation Myocardial Infarction (STEMI) and their different effect on myocardial perfusion and in-hospital Major Adverse Cardiac Events. Methods: The study included 170 patients with Type II diabetes, who presented with acute anterior wall STEMI who underwent primary PCI. They were randomized into two groups, the $1^{\text {st }}$ group 85 patients received clopidogrel loading dose (600 mg) and the $2^{\text {nd }}$ group 85 patients received ticagrelor loading dose $(180 \mathrm{mg})$. Postinterventional thrombolysis in myocardial infarction (TIMI) flow grade and myocardial blush grade (MBG) were recorded as well as in-hospital outcomes. Results: In the clopidogrel group, $80 \%$ of the patients had TIMI III flow score, $15.3 \%$ had TIMI II flow score and 2.4\% had TIMI I and TIMI 0 flow score. In the ticagrelor group, $95 \%$ of the patients had TIMI III flow score, $2.4 \%$ had TIMI II flow score and 1.2\% had TIMI I and TIMI 0 flow score $(P=0.01)$. Regarding MBG, in the clopidogrel group, $64.7 \%$ of the patients had MBG III, 13\% had MBG II flow score, 2.3\% had MBG 1, and 20\% had MBG 0 . In the ticagrelor group, 93\% of the patients had MBG III, 1.1\% had MBG II flow score, 1.1\% had MBG 1, and 4.8\% had MBG $0(P=0.007)$. In the clopidogrel group there were $3.5 \%$ in-hospital mortality, while in ticagrelor $2.4 \%$ in-hospital mortality $(2.4 \%)(P=0.47)$. In the Clopidogrel group, in-hospital MACE was $5.8 \%$ versus $3.5 \%$ in the ticagrelor group $(P=0.65)$. In the Clopidogrel group, in-hospital bleeding was $3.5 \%$ versus $4.7 \%$ in the ticagrelor group $(P=0.7)$. Conclusion: Ticagrelor loading before primary PCI resulted in improved TIMI flow and MBG in Type II diabetic patients presenting with anterior wall myocardial infarction.

Keywords: Blush grade, clopidogrel, no-reflow, primary percutaneous coronary intervention, ticagrelor, thrombolysis in myocardial infarction flow

\section{INTRODUCTION}

Coronary artery disease is the most important cause of death worldwide. ${ }^{[1]}$ ST-segment elevation myocardial infarction (STEMI) is a significant cause of cardiovascular morbidity and mortality. Successful treatment depends on rapid successful reperfusion therapy. ${ }^{[2]}$ Primary percutaneous coronary intervention $(\mathrm{PCI})$ is the preferred reperfusion strategy

Received: 14-11-2020 Revised: 09-12-2020 Accepted: 26-04-2021

Published Online: 05-07-2021

\begin{tabular}{|l|l|}
\hline \multicolumn{3}{|c|}{ Access this article online } \\
\hline Quick Response Code: & Website: \\
& \\
&
\end{tabular}

for patients presenting with STEMI, however periprocedural myocardial injury and coronary no-reflow (CNR) can still occur and result in worse in-hospital and long-term outcomes. CNR

Address for correspondence: Dr. Emad Effat Fakhry, 16 Abd El Salam Basha Zohny Gamea El Fath Heliopolis, Cairo, Egypt. E-mail: effat@med.asu.edu.eg

This is an open access journal, and articles are distributed under the terms of the Creative Commons Attribution-NonCommercial-ShareAlike 4.0 License, which allows others to remix, tweak, and build upon the work non-commercially, as long as appropriate credit is given and the new creations are licensed under the identical terms.

For reprints contact: WKHLRPMedknow_reprints@wolterskluwer.com

How to cite this article: Fakhry EE, El Etriby AM, Nayel AE. Comparison between preoperative loading dose of ticagrelor versus clopidogrel on myocardial perfusion in type II diabetic patients presenting with anterior wall myocardial infarction. Int J Cardiovasc Acad 2021;7:55-9. 
is associated with more extensive myocardial injury, worse segmental and global left ventricular contractility, malignant arrhythmias, and higher mortality. ${ }^{[3]}$ Diabetes mellitus was recognized as an independent risk factor for the development of CNR,${ }^{[4]}$ thus diabetic patients are at higher risk of developing cardiovascular complications. Ticagrelor significantly reduced the rate of death from myocardial infarction, vascular causes, or stroke without an increased rate of major bleeding as reported by the PLATO trial. ${ }^{[5]}$ Ticagrelor increases the circulatory level of adenosine, which may decrease the microcirculatory resistance and that may be protective against no-reflow. The aim of this study is to compare the preoperative loading dose of Ticagrelor versus Clopidogrel on myocardial perfusion in Type II diabetic patients with anterior STEMI undergoing primary PCI.

\section{Methods}

The study was conducted on 170 patients with Type II Diabetes mellitus presenting anterior STEMI who underwent primary PCI. Patients were randomized into two groups ( 85 patients each) according to the loading dose of P2Y12 inhibitor; $1^{\text {st }}$ group received a clopidogrel loading dose $(600 \mathrm{mg})$ and the $2^{\text {nd }}$ group received ticagrelor loading dose $(180 \mathrm{mg})$. Postinterventional thrombolysis in myocardial infarction (TIMI) flow grade and myocardial blush grade (MBG) were recorded during the procedure and compared as the primary endpoint, and the patients were followed up for in-hospital outcomes (including death, MACE, and bleeding) as a secondary endpoint.

Patients presenting with STEMI after $48 \mathrm{~h}$ from the onset of chest pain, those who received thrombolytic therapy, patients with hematological disorders, acute inflammatory diseases, hepatic failure, cancer, chronic renal disease on a hemodialysis program and patients with known allergy or intolerance to statin therapy or previously on statin therapy all were excluded from the study.

The study was approved by the Research Ethics Committee, and all patients signed informed written consent for participation in the study in accordance with the declaration of Helsinki. All patients were subjected to: (1) Detailed medical history and complete clinical examination (2) 12 lead surface ECG before and after primary PCI to determine the extent of ST-segment resolution (STR) calculated as the sum of ST-segment elevation on initial Electrocardiography (ECG) minus the sum of ST-segment elevation on the ECG at 90 min after PCI, divided by the sum of ST-segment elevation on the initial ECG, and was presented as a percentage. The complete early STR was defined as $\geq 70 \%$ STR. (3) Coronary angiography was done, patients with preoperative TIMI 0 grade and preoperative TIMI Thrombus Grade 5 (total occlusion) were included in the study while those with higher preoperative TIMI flow grade or with TIMI thrombus grade $<5$ were excluded from the study. All patients received $300 \mathrm{mg}$ Aspirin as a loading dose at the time of admission and Enoxaparin $0.5 \mathrm{mg} / \mathrm{kg}$ intravenous as a preantral anticoagulant at the time of the procedure.
Patients who received Aspiration therapy, Glycoprotein IIb/ IIIa inhibitors, Morphine were excluded from the study. After primary PCI TIMI flow score and TIMI MBG were identified. (4) Transthoracic echocardiography: Routine echo study will be performed with special emphasis on ejection fraction measured by the modified Simpson method by expert operators blinded from the study protocol using a GE Vivid E95 machine (5) In-Hospital death, bleeding, and MACE such as nonfatal stroke, nonfatal myocardial infarction, and cardiovascular death.

Data were collected and entered into the Statistical Package for the Social Science (IBM SPSS) version 20, USA. The data were presented in the form of numbers and percentages for the qualitative data, mean, standard deviations, and ranges for the quantitative data with parametric distribution Chi-square test was used to in comparison of qualitative data between the two groups. The comparison between two groups with quantitative data and parametric distribution was done by using Independent $t$-test. The confidence interval was set to $95 \%$ and the margin of error accepted was set to $5 \%$.

\section{Ethical statement}

The study was approved by the Research Ethics Committee (Faculty Of Medicine, Ain Shams University, FWA 0006444), and all patients signed informed written consent for participation in the study in accordance with the declaration of Helsinki.

\section{RESULTS}

This study was conducted on 170 patients with Type II Diabetes mellitus presented with anterior STEMI who underwent primary PCI. Patients were divided into two groups; the first group included the patients who received a loading dose of clopidogrel. The second group included patients who received a loading dose of ticagrelor. Regarding baseline characteristics, there was no statistically significant difference between the 2 groups as regards age, gender, smoking, body mass index, history of hypertension, previous history of ischemic heart disease, chronic obstructive pulmonary disease, Previous PCI, previous coronary artery bypass graft surgery, history of Cerebrovascular stroke, Receiving Aspirin before the incident event, peripheral vascular disease, serum creatinine level, hemoglobin level, Killip class, and door to balloon time [Table 1].

As regards the procedural details, there were no statically significant differences between the two groups regarding the site of total occlusion, predilatation, postdilatation, length of the stent, diameter of the stents, number of the stents implanted [Table 2].

In the clopidogrel group, $80 \%$ of the patients had TIMI III flow, $15.3 \%$ had TIMI II flow, $2.3 \%$ had TIMI I and $2.3 \%$ had TIMI 0 flow. In the ticagrelor group, $95 \%$ of the patients had TIMI III flow, $2.4 \%$ had TIMI II flow, $1.2 \%$ had TIMI I, and $1.2 \%$ had TIMI I flow, with a significant $P=0.01$. 


\begin{tabular}{|c|c|c|c|}
\hline & \multicolumn{3}{|c|}{$n(\%)$} \\
\hline Age, mean \pm SD (range) & \multicolumn{3}{|c|}{$55.58 \pm 10.19(31-80)$} \\
\hline Male gender & \multicolumn{3}{|c|}{$117(68.8)$} \\
\hline Smoker & \multicolumn{3}{|c|}{119 (70.0) } \\
\hline $\mathrm{BMI}\left(\mathrm{kg} / \mathrm{m}^{2}\right)$, mean $\pm \mathrm{SD}$ (range) & \multicolumn{3}{|c|}{$27 \pm 5(18-35)$} \\
\hline HTN & \multicolumn{3}{|c|}{$44(25.9)$} \\
\hline History of IHD & \multicolumn{3}{|c|}{$22(12.9)$} \\
\hline COPD & \multicolumn{3}{|c|}{$35(20.6)$} \\
\hline Pervious PCI & \multicolumn{3}{|c|}{$34(20)$} \\
\hline Previous CABG & \multicolumn{3}{|c|}{$4(2.4)$} \\
\hline History of CVS & \multicolumn{3}{|c|}{$17(10)$} \\
\hline Receiving aspirin before incident event & \multicolumn{3}{|c|}{$50(29.4)$} \\
\hline Peripheral vascular disease & \multicolumn{3}{|c|}{$36(21.2)$} \\
\hline \multicolumn{4}{|l|}{ Killip class } \\
\hline I & \multicolumn{3}{|c|}{$153(90)$} \\
\hline II & \multicolumn{3}{|c|}{$10(5.9)$} \\
\hline III & \multicolumn{3}{|c|}{$4(2.4)$} \\
\hline \multirow[t]{2}{*}{ IV } & \multicolumn{3}{|c|}{$3(1.8)$} \\
\hline & Ticagrelor group ( $n=85), n(\%)$ & Clopidogrel group ( $n=85), n$ (\%) & $P$ \\
\hline Age, mean \pm SD (range) & $55.27 \pm 10.30(31-80)$ & $55.89 \pm 10.13(34-79)$ & 0.691 \\
\hline Male gender & $56(65.9)$ & $61(71.8)$ & 0.408 \\
\hline Smoker & $63(74.1)$ & $56(65.9)$ & 0.241 \\
\hline Hypertension & $22(25.9)$ & $22(25.9)$ & 1.00 \\
\hline History of IHD & $10(11.8)$ & $12(14.1)$ & 0.648 \\
\hline COPD & $19(22.4)$ & $16(18.8)$ & 0.569 \\
\hline Previous PCI & $16(18.8)$ & $18(21.2)$ & 0.384 \\
\hline Previous $\mathrm{CABG}$ & $2(2.35)$ & $2(2.35)$ & 1 \\
\hline History of CVS & $8(9.4)$ & $9(10.6)$ & 0.795 \\
\hline Receiving aspirin before incident event & $28(32.9)$ & $22(25.9)$ & 0.313 \\
\hline Peripheral vascular disease & $19(22.4)$ & $17(20)$ & 0.704 \\
\hline Serum creatinine level (mg/dl) & $1.2 \pm 0.3$ & $1.1 \pm 0.2$ & 0.552 \\
\hline Hemoglobin level (g/dl) & $12.2 \pm 3.1$ & $14 \pm 2.2$ & 0.321 \\
\hline \multicolumn{4}{|l|}{ Killip class } \\
\hline I & $78(91.8)$ & $75(88.2)$ & 0.441 \\
\hline II & $5(5.9)$ & $6(7.1)$ & 0.757 \\
\hline III & $2(2.4)$ & $2(2.4)$ & 1 \\
\hline IV & $2(2.4)$ & $1(1.2)$ & 0.562 \\
\hline Door to balloon time (min) & $58 \pm 16$ & $60 \pm 12$ & 0.357 \\
\hline
\end{tabular}

Regarding $\mathrm{MBG}$, in the clopidogrel group, $64.7 \%$ of the patients had MBG III, 13\% had MBG II, 2.3\% had MBG I and $20 \%$ had MBG 0 . In the ticagrelor group, $93 \%$ of the patients had MBG III, 1.1\% had MBG II, 1.1\% had MBG 1 and $4.8 \%$ had MBG 0 , with a significant $P=0.007$.

No reflow occurred in $22.4 \%$ in the clopidogrel group and $5.8 \%$ in the ticagrelor group with a significant $P=0.002$ [Table 3].

Regarding in-hospital death $3.5 \%$ of patients in the clopidogrel group died during the in-hospital stay versus $2.4 \%$ in the ticagrelor group, $P=0.468$, Regarding in-hospital MACE occurred in $5.8 \%$ of patients in the clopidogrel group versus $3.5 \%$ in the ticagrelor group, $P=0.65$, Regarding in-hospital bleeding occurred in $3.5 \%$ of patients in the clopidogrel group versus $4.7 \%$ in the ticagrelor group, $P=0.700$ [Table 4$]$.

\section{Discussion}

STEMI is a leading cause of morbidity and mortality. Primary PCI is the recommended therapy for STEMI. No-reflow is an independent predictor of in-hospital mortality. ${ }^{[6]}$ No-reflow refers to myocardial under-perfusion in the absence of mechanical obstruction. CNR is associated with worse left ventricular contractility, more extensive myocardial necrosis, malignant arrhythmias, and increased mortality. ${ }^{[3]}$ Type 2 diabetes mellitus is associated with poorer outcomes in coronary artery disease than nondiabetic counterparts after 


\begin{tabular}{|c|c|c|c|}
\hline & $\begin{array}{l}\text { Clopidogrel } \\
\text { group }\end{array}$ & $\begin{array}{l}\text { Ticagrelor } \\
\text { group }\end{array}$ & $P$ \\
\hline \multicolumn{4}{|l|}{ Site of total occlusion } \\
\hline Left main & 1 & 0 & 0.451 \\
\hline Proximal LAD & 60 & 55 & \\
\hline Mid LAD & 24 & 29 & \\
\hline Distal LAD & 0 & 1 & \\
\hline Predilatation & 60 & 63 & 0.512 \\
\hline Postdilatation & 14 & 19 & 0.381 \\
\hline Stent length (mm) & $28.1 \pm 7$ & $26.3 \pm 8$ & 0.715 \\
\hline Stent diameter $($ mean $\pm \mathrm{SD})$ & $3.2 \pm 0.5$ & $3.1 \pm 0.7$ & 0.802 \\
\hline \multicolumn{4}{|l|}{ Number of stents } \\
\hline One stent & 50 & 52 & 0.750 \\
\hline More than one stent & 35 & 33 & \\
\hline
\end{tabular}

Patients who implanted more than one stent the length of the stent was taken as a sum of the length of the stents implanted, and the diameter was taken as the diameter of the largest stent. No statistically significant difference between the two groups as regards procedural details. $\mathrm{LAD}=\mathrm{Left}$ anterior descending artery; $\mathrm{SD}=$ Standard deviation

\begin{tabular}{|c|c|c|c|}
\hline & $\begin{array}{l}\text { Clopidogrel } \\
\text { group, } n(\%)\end{array}$ & $\begin{array}{c}\text { Ticagrelor } \\
\text { group, } \boldsymbol{n}(\%)\end{array}$ & $P$ \\
\hline \multicolumn{4}{|c|}{ TIMI flow } \\
\hline 0 & $2(2.35)$ & $1(1.2)$ & 0.01 \\
\hline I & $2(2.35)$ & $1(1.2)$ & \\
\hline II & $13(15.3)$ & $2(2.4)$ & \\
\hline III & $68(80.0)$ & $81(95.2)$ & \\
\hline \multicolumn{4}{|c|}{ MBG } \\
\hline 0 & $17(20)$ & $4(4.8)$ & 0.007 \\
\hline I & $2(2.3)$ & $1(1.1)$ & \\
\hline II & $11(13)$ & $1(1.1)$ & \\
\hline III & $55(64.7)$ & $79(93)$ & \\
\hline
\end{tabular}

$\begin{array}{llll}\text { No reflow } & 19(22.4) & 5(5.8) & 0.002\end{array}$

TIMI flow grade, MBG were significantly higher in the ticagrelor group and the incidence of no reflow was lower in the ticagrelor group in comparison to clopidogrel group. $\mathrm{MBG}=$ Myocardial blush grade; TIMI flow=Thrombolysis in myocardial infarction flow

\begin{tabular}{|c|c|c|c|}
\hline & $\begin{array}{l}\text { Clopidogrel } \\
\text { group, } n(\%)\end{array}$ & $\begin{array}{c}\text { Ticagrelor } \\
\text { group, } \boldsymbol{n}(\%)\end{array}$ & $P$ \\
\hline Death & $3(3.5)$ & $3(3.5)$ & 0.468 \\
\hline MACE & $5(5.8)$ & $5(5.8)$ & 0.650 \\
\hline Bleeding & $3(3.5)$ & $3(3.5)$ & 0.700 \\
\hline
\end{tabular}

There were no statistically significant differences between the two groups as regards in hospital death, MACE and bleeding, MACE $=$ Major adverse cardiac events

coronary intervention. ${ }^{[7]}$ This might be related to vessel wall inflammation, endothelial dysfunction, and the development of vulnerable plaques liable for distal embolization. We studied the difference between preoperative loading dose of Ticagrelor versus Clopidogrel on myocardial perfusion in type II Diabetic patients presenting with anterior wall myocardial infarction. Ticagrelor loading before primary PCI resulted in improved TIMI flow and MBG in this population. Ticagrelor inhibits cellular uptake of adenosine and thus, increasing the circulating adenosine levels. Adenosine has a potent vasodilator, anti-inflammatory, and antiplatelet effect that may protect the myocardium from both ischemic and reperfusion injuries ${ }^{[8]}$ and this might explain our results. Kim EK et al. showed that the Infarct size in STEMI patients assessed by cardiac MRI was reduced in patients receiving Ticagrelor versus clopidogrel. This protective effect was independent of ticagrelor-induced platelet inhibition ${ }^{[9]}$ In the CV-TIME trial ticagrelor loading dose reduced microvascular injury in comparison with a loading dose of clopidogrel, as assessed by the index of microcirculatory resistance immediately after primary PCI. ${ }^{[10]}$ Wang et al. demonstrated a higher MBG, lower corrected TIMI frame count, and a higher rate of complete STR with Ticagrelor in comparison to the clopidogrel. ${ }^{[11]}$ Lui et al. showed that the use of ticagrelor in diabetic patients presenting with STEMI improves the level of myocardial microcirculation perfusion and improves the left ventricular function. ${ }^{[12]}$

Contradictory results from the MICAMI-TICLO trial showed that ticagrelor did not significantly improve angiographic or electrocardiographic parameters of myocardial reperfusion in STEMI patients, in comparison to clopidogrel. ${ }^{[13]}$ Di Vito et al. showed that TIMI flow, TIMI frame count, and STR were comparable between clopidogrel and ticagrelor groups. ${ }^{[14]}$ These different results may be due to different populations included in different studies were in our study we included exclusively patients with Type II diabetes mellitus. Moreover, commonly co-treatment with morphine may impair the rate of absorption of $\mathrm{P} 2 \mathrm{Y} 12$ inhibitors, including ticagrelor in patients with STEMI. ${ }^{[15]}$ This may deprive the ticagrelor of its rapid onset of action.

In our study, the rate of in-hospital death and myocardial infarction was lower in the ticagrelor group although it did not reach statistical significance. In the sub-study of the PLATO trial that includes only diabetic patients a significant reduction in the 30-day rates of death from any cause and myocardial infarction in patients with the acute coronary syndrome. ${ }^{[16]}$

\section{Conclusion}

Ticagrelor loading before primary PCI resulted in improved TIMI flow and MBG in Type II diabetic patients presenting with anterior wall myocardial infarction.

\section{Acknowledgments}

Technicians and nursing teams of Ain shams university Cath-lab.

\section{Financial support and sponsorship}

Nil. 


\section{Conflicts of interest}

There are no conflicts of interest.

\section{Declaration of patient consent}

The authors certify that they have obtained all appropriate patient consent forms. In the form, the patients have given their consent for their images and other clinical information to be reported in the journal. The patient understands that name and initials will not be published and due efforts will be made to conceal identity, but anonymity cannot be guaranteed.

\section{RefERENCES}

1. Townsend N, Wilson L, Bhatnagar P, Wickramasinghe K, Rayner M, Nichols M. Cardiovascular disease in Europe: Epidemiological update 2016. Eur Heart J 2016;37:3232-45.

2. Choudhury T, West NE, El-Omar M. ST elevation myocardial infarction. Clin Med (Lond) 2016;16:277-82.

3. Morishima I, Sone T, Okumura K, Tsuboi H, Kondo J, Mukawa H, et al. Angiographic no-reflow phenomenon as a predictor of adverse long-term outcome in patients treated with percutaneous transluminal coronary angioplasty for first acute myocardial infarction. J Am Coll Cardiol 2000;36:1202-9.

4. Nair Rajesh G, Jayaprasad N, Madhavan S, Sudha Kumary V, Jayaprakash K, Raihanathul Misiriya KJ, et al. Predictors and prognosis of no-reflow during primary percutaneous coronary intervention. Proc (Bayl Univ Med Cent) 2019;32:30-3.

5. Wallentin L, Becker RC, Budaj A, Cannon CP, Emanuelsson H, Held C, et al. Ticagrelor versus clopidogrel in patients with acute coronary syndromes. N Engl J Med 2009;361:1045-57.

6. Resnic FS, Wainstein M, Lee MK, Behrendt D, Wainstein RV, Ohno-Machado L, et al. No-reflow is an independent predictor of death and myocardial infarction after percutaneous coronary intervention. Am Heart J 2003;145:42-6.

7. Machecourt J, Danchin N, Lablanche JM, Fauvel JM, Bonnet JL, Marliere S, et al. Risk factors for stent thrombosis after implantation of sirolimus-eluting stents in diabetic and nondiabetic patients:
The EVASTENT Matched-Cohort Registry. J Am Coll Cardiol 2007;50:501-8.

8. Cattaneo M, Schulz R, Nylander S. Adenosine-mediated effects of ticagrelor: Evidence and potential clinical relevance. J Am Coll Cardiol 2014;63:2503-9.

9. Kim EK, Park TK, Yang JH, Song YB, Choi JH, Choi SH, et al. Ticagrelor versus clopidogrel on myocardial infarct size in patients undergoing primary percutaneous coronary intervention. J Am Coll Cardiol 2017;69:2098-9.

10. Park SD, Lee MJ, Baek YS, Kwon SW, Shin SH, Woo SI, et al. Randomised trial to compare a protective effect of Clopidogrel Versus TIcagrelor on coronary Microvascular injury in ST-segment Elevation myocardial infarction (CV-TIME trial). EuroIntervention 2016;12:e964-e971.

11. Wang X, Li X, Wu H, Li R, Liu H, Wang L, et al. Beneficial effect of ticagrelor on microvascular perfusion in patients with ST-segment elevation myocardial infarction undergoing a primary percutaneous coronary intervention. Coron Artery Dis 2019;30:317-22.

12. Liu Y, Ding LY, Li XZ. Therapy with ticagrelor for ST-elevated acute coronary syndrome accompanied by diabetes mellitus. Eur Rev Med Pharmacol Sci 2019;23:312-8.

13. Winter JL, Lindefjeld DS, Veas N, Guarda E, Valdebenito M, Méndez M, et al. Angiographic and electrocardiographic parameters of myocardial reperfusion in angioplasty of patients with ST elevation acute myocardial infarction loaded with ticagrelor or clopidogrel (MICAMI-TICLO trial). Cardiovasc Revasc Med 2014;15:284-8.

14. Di Vito L, Versaci F, Limbruno U, Pawlowski T, Gatto L, Romagnoli E, et al. Impact of oral P2Y12 inhibitors on residual thrombus burden and reperfusion indexes in patients with ST-segment elevation myocardial infarction. J Cardiovasc Med (Hagerstown) 2016;17:701-6.

15. Parodi G, Valenti R, Bellandi B, Migliorini A, Marcucci R, Comito V, et al. Comparison of prasugrel and ticagrelor loading doses in ST-segment elevation myocardial infarction patients: RAPID (Rapid Activity of Platelet Inhibitor Drugs) primary PCI study. J Am Coll Cardiol 2013;61:1601-6.

16. James S, Angiolillo DJ, Cornel JH, Erlinge D, Husted S, Kontny F, et al. Ticagrelor vs. clopidogrel in patients with acute coronary syndromes and diabetes: A substudy from the PLATelet inhibition and patient Outcomes (PLATO) trial. Eur Heart J 2010;31:3006-16. 